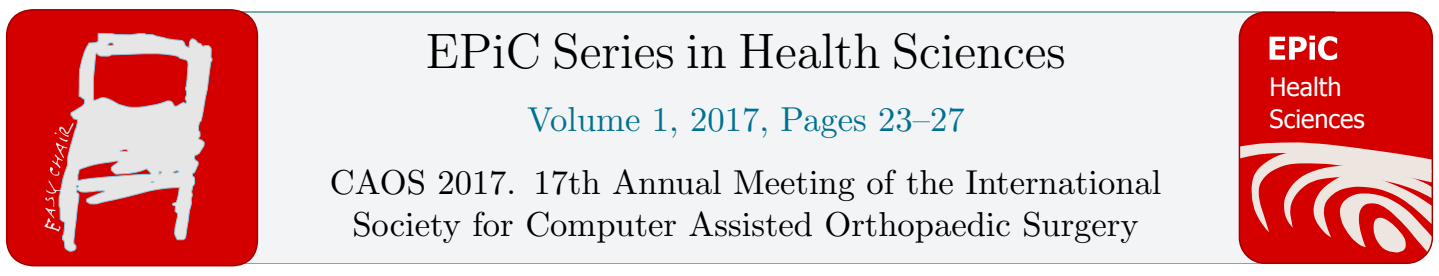

\title{
Pedicle screw insertion in spine: a randomized controlled study for robot-assisted spinal surgery
}

\author{
Wei Tian ${ }^{1 *}$, Mingxing Fan $^{2}$ and Yajun $\mathrm{Liu}^{2}$ \\ 1*: Department of Spine surgery, Beijing Jishuitan Hospital, Beijing, 100035, China, \\ 2: Department of Spine surgery, Beijing Jishuitan Hospital, Beijing, 100035, China \\ drtianwei a @163.com
}

\begin{abstract}
To introduce a new robot-assisted surgical system for spinal posterior fixation which called TiRobot, based on intraoperative three-dimensional images. TiRobot has three components: the planning and navigation system, optical tracking system and robotic arm system. By combining navigation and robot techniques, TiRobot can guide the screw trajectories for orthopedic surgeries.

In this randomized controlled study approved by the Ethics Committee, 40 patients were involved and all has been fully informed and sign the informed consent. 17 patients were treated by free-hand fluoroscopy-guided surgery, and 23 patients were treated by robot-assisted spinal surgery.

A total of 190 pedicle screws was implanted. The overall operation time were not different for both groups. None of the screws necessitated re-surgery for revised placement. In the robot-assisted group, assessment of pedicle screw accuracy showed that 102 of 102 screws $(100 \%)$ were safely placed $(<2 \mathrm{~mm}$, category $\mathrm{A}+\mathrm{B})$. And mean deviation in entry point was $1.70+/-0.83 \mathrm{~mm}$, mean deviation in end point was $1.84+/$ $1.04 \mathrm{~mm}$. In the conventional freehand group, assessment of pedicle screw accuracy showed that 87 of $88(98.9 \%)$ were safely placed $(<2 \mathrm{~mm}$, category A+B), 1 screw fall in category $\mathrm{C}$, mean deviation in entry point was $3.73+/-2.28 \mathrm{~mm}$, mean deviation in end point was $4.11+/-2.31 \mathrm{~mm}$.

This randomized controlled study verified that robot-assisted pedicle screw placement with real-time navigation is a more accuracy and safer method, also revealed great clinical potential of robot-assisted surgery in the future.
\end{abstract}

K. Radermacher and F. Rodriguez Y Baena (eds.), CAOS 2017 (EPiC Series in Health Sciences, vol. 1), pp. $23-27$ 


\section{Introduction}

Pedicle screw placement is an advanced posterior fixation technic that could have substantial biomechanical benefits for patients[1]. However, anatomical variations of vertebrae are wide, and the region is adjacent to important organ such as spinal cords, dura and arteries, all of which make the fixation harder. Computer-assisted navigation has been proved to improve the screw placement accuracy[2], but it may need surgeon repeatedly adjust the placement trajectories, which is nonconvenience[3]. Robot-assisted navigation can make up for the above shortcomings, and has the potential to more improve the screw placement accuracy. In recent days, we designed a new robot system combined with navigation, and which we believe will make spinal surgery more accuracy and safer.

\section{Materials and methods}

The recently new designed robot system was called TiRobot, which is based on intraoperative three-dimensional images. TiRobot has three components: the planning and navigation system, optical tracking system and robotic arm system. A set of intraoperative images is initially acquired using a 3D C-arm (Siemens Medical Solutions, Erlangen, Germany). The robotic arm is identified at the same time by detecting the plate locator on it. After that, the planning and navigation system can perform an automatic registration. Surgeons then plan the entry point and trajectory of the screw on the system and send these to guide the robotic arm. The optical tracking system is made up of an infrared stereo camera and two reference frames, one attached to the spinous process and the other to the robotic arm, thus enabling the optical tracking system to locate the position of the robotic arm relative to the patient and to guide the arm to the planned position. The robotic arm system is built on a mobile platform and has six degree of freedom. It has universal holders on which the reference frame, plate locator, guide holder and surgical instruments can be mounted. The robotic arm spontaneously moves accurately to the required position under the guidance of the planning and navigation and optical tracking systems. By combining navigation and robot techniques, TiRobot can guide the screw trajectories for orthopedic surgeries.

In this randomized controlled study which was approved by the Ethics Committee, 40 patients were involved and all has been fully informed and sign the informed consent. 17 patients were treated by free-hand fluoroscopy-guided surgery, and 23 patients were treated by robot-assisted spinal surgery. After surgery, the operation time of two groups were compared, further, a postoperative CT scan was performed. The CT image data were reconstructed in sagittal, coronal and axial views by the mimics 15.0 software, and a blind evaluation of the position of screws was performed by two spine surgeons who were not involved in the surgery. Any penetration of the cortex in the lateral, medial, cranial or caudal directions was measured according to the Gertzbein-Robbins classification[4] (A: no cortical violation; B: cortical breach $<2 \mathrm{~mm}$; C: $\geqslant 2 \mathrm{~mm}$ to $<4 \mathrm{~mm}$; D: $\geqslant 4 \mathrm{~mm}$ to $<6 \mathrm{~mm}$; E: $\geqslant 6$ $\mathrm{mm})$. Also, we measured the discrepancies between the actual path and planned trajectory in entry point and angle deviation[5]. 


\section{Results}

A total of 190 pedicle screws were implanted in 40 patients. There were no differences between these two groups in the baseline characteristics. Preparation time in the operating room, overall operation time were not different for both groups (138.9 +/- $46.6 \mathrm{~min}$ in robot-assisted group compared with $118.2+/-40.6 \mathrm{~min}$ in conventional freehand group). None of the screws necessitated re-surgery for revised placement. In the robot-assisted group, assessment of pedicle screw accuracy showed that 102 of 102 screws $(100 \%)$ were safely placed $(<2 \mathrm{~mm}$, category A+B), i.e., good screw positions (Fig. 1). And mean deviation in entry point was $1.70+/-0.83 \mathrm{~mm}$, mean deviation in end point was $1.84+/-1.04 \mathrm{~mm}$, mean difference in screw insertion was $1.77+/-0.78 \mathrm{~mm}$. In the conventional freehand group, assessment of pedicle screw accuracy showed that 87 of 88 (98.9\%) were safely placed $(<2 \mathrm{~mm}$, category $\mathrm{A}+\mathrm{B}), 1$ screw fall in category $\mathrm{C}$, mean deviation in entry point was $3.73+/-2.28 \mathrm{~mm}$, mean deviation in end point was $4.11+/-2.31 \mathrm{~mm}$, mean difference in screw insertion was $3.92+/-1.80 \mathrm{~mm}$ (Table 1 ).
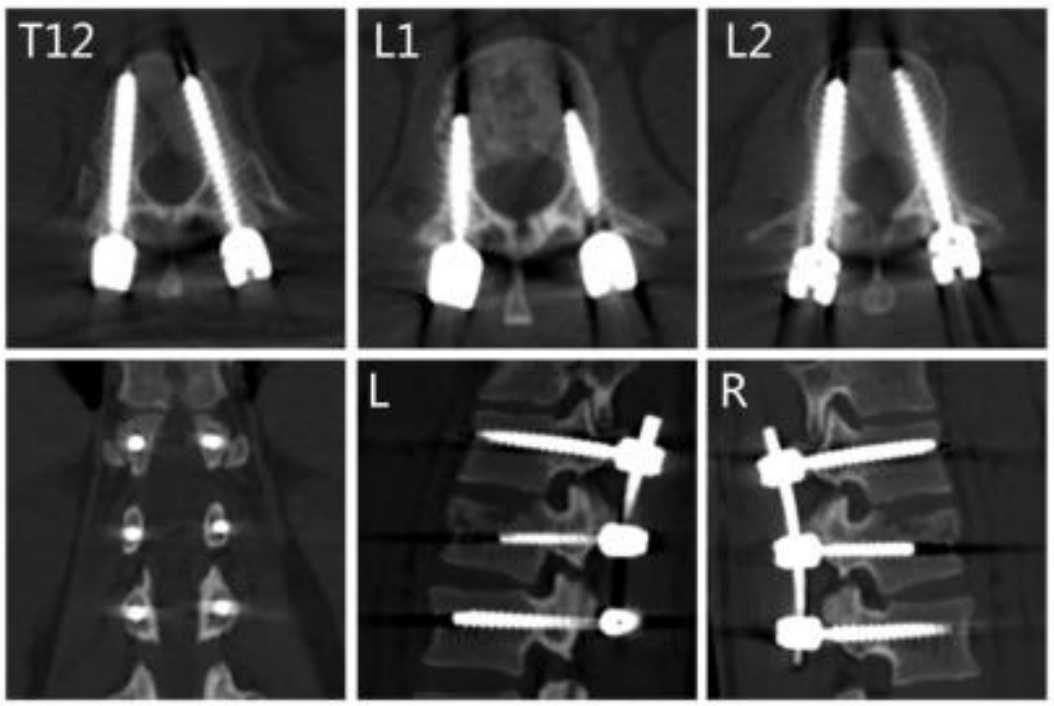

Figure 1: Postoperative computer tomography scans indicating the results of the thoracic and lumber screws for safety analysis.

\begin{tabular}{|c|c|c|c|c|}
\hline & $\begin{array}{c}\text { Overall } \\
\text { surgery time }\end{array}$ & $\begin{array}{c}\text { Entry-point } \\
\text { deviation }(\mathrm{mm})\end{array}$ & $\begin{array}{c}\text { End-point } \\
\text { deviation }(\mathrm{mm})\end{array}$ & $\begin{array}{l}\text { Mean deviation } \\
(\mathrm{mm})\end{array}$ \\
\hline $\begin{array}{c}\text { Robot- } \\
\text { assisted group }\end{array}$ & $138.9 \pm 46.6$ & $1.70 \pm 0.83$ & $1.84 \pm 1.04$ & $1.77 \pm 0.78$ \\
\hline $\begin{array}{l}\text { Conventional } \\
\text { freehand } \\
\text { group }\end{array}$ & $118.2 \pm 40.6$ & $3.73 \pm 2.28$ & $4.11 \pm 2.31$ & $3.92 \pm 1.80$ \\
\hline $\mathrm{P}$ value & 0.557 & $\mathrm{p}<0.001$ & $\mathrm{p}<0.001$ & $\mathrm{p}<0.001$ \\
\hline
\end{tabular}


Table 1: Comparison of surgical time and the accuracy of the inserted screws between robotassisted group and conventional freehand group

\section{Discussions}

Screw fixation is the fundamental process in all spinal surgery, however, screws fixation can be technically challenging because of the difficult approach and complex morphology of the vertebrae and surrounding vital structures. Surgeons performing this procedure are exposed to high irradiation doses yet have still failed to achieve satisfactory safety and accuracy, the reported misplacement rate of the pedicle screws is approximately $4.9 \%$ to $37.5 \%[6-12]$. Traditional methods as well as navigation methods for intraoperative spinal localization play an important role in spinal surgery. However, these methods do not show the great advantages, which have led to the use of robot-assisted surgery. Many studies focused on this field in recent years, such as the SpineAssit/Renaissance, SPINEBOT, VectorBot and Neuroglide[13], however, most of them are still based on fluoroscopyguided navigation but not $3 \mathrm{D}$ real-time navigation.

In response, we designed the TiRobot with real-time 3D navigation, which could provide accurate positioning, adequate steadiness and repeatability. And this randomized controlled study verified that robot-assisted pedicle screw placement with real-time 3D navigation is a more accuracy and safer method than conventional way, and also revealed great clinical potential of robot-assisted surgery in the future.

\section{References}

[1] A.H. Barnes, J.A. Eguizabal, F.L. Acosta, Jr., J.C. Lotz, J.M. Buckley, C.P. Ames, Biomechanical pullout strength and stability of the cervical artificial pedicle screw, Spine 34(1) (2009) E16-20.

[2] T. Tjardes, S. Shafizadeh, D. Rixen, T. Paffrath, B. Bouillon, E.S. Steinhausen, H. Baethis, Image-guided spine surgery: state of the art and future directions, European spine journal : official publication of the European Spine Society, the European Spinal Deformity Society, and the European Section of the Cervical Spine Research Society 19(1) (2010) 25-45.

[3] R. Verma, S. Krishan, K. Haendlmayer, A. Mohsen, Functional outcome of computer-assisted spinal pedicle screw placement: a systematic review and meta-analysis of 23 studies including 5,992 pedicle screws, European spine journal : official publication of the European Spine Society, the European Spinal Deformity Society, and the European Section of the Cervical Spine Research Society 19(3) (2010) 370-5.

[4] I. Pechlivanis, G. Kiriyanthan, M. Engelhardt, M. Scholz, S. Lucke, A. Harders, K. Schmieder, Percutaneous placement of pedicle screws in the lumbar spine using a bone mounted miniature robotic system: first experiences and accuracy of screw placement, Spine 34(4) (2009) 392-8. 
[5] S. Kim, J. Chung, B.J. Yi, Y.S. Kim, An assistive image-guided surgical robot system using Oarm fluoroscopy for pedicle screw insertion: preliminary and cadaveric study, Neurosurgery 67(6) (2010) 1757-67; discussion 1767.

[6] W. Tian, Y. Liu, S. Zheng, Y. Lv, Accuracy of lower cervical pedicle screw placement with assistance of distinct navigation systems: a human cadaveric study, European spine journal : official publication of the European Spine Society, the European Spinal Deformity Society, and the European Section of the Cervical Spine Research Society 22(1) (2013) 148-55.

[7] N.F. Tian, Q.S. Huang, P. Zhou, Y. Zhou, R.K. Wu, Y. Lou, H.Z. Xu, Pedicle screw insertion accuracy with different assisted methods: a systematic review and meta-analysis of comparative studies, European spine journal : official publication of the European Spine Society, the European Spinal Deformity Society, and the European Section of the Cervical Spine Research Society 20(6) (2011) 846-59.

[8] D.P. Devito, L. Kaplan, R. Dietl, M. Pfeiffer, D. Horne, B. Silberstein, M. Hardenbrook, G. Kiriyanthan, Y. Barzilay, A. Bruskin, D. Sackerer, V. Alexandrovsky, C. Stuer, R. Burger, J. Maeurer, G.D. Donald, R. Schoenmayr, A. Friedlander, N. Knoller, K. Schmieder, I. Pechlivanis, I.S. Kim, B. Meyer, M. Shoham, Clinical acceptance and accuracy assessment of spinal implants guided with SpineAssist surgical robot: retrospective study, Spine 35(24) (2010) 2109-15.

[9] V. Kosmopoulos, C. Schizas, Pedicle screw placement accuracy: a meta-analysis, Spine 32(3) (2007) E111-20.

[10] S. Burch, Surgical complications of spinal deformity surgery, Neurosurgery clinics of North America 18(2) (2007) 385-92.

[11] L.T. Holly, K.T. Foley, Three-dimensional fluoroscopy-guided percutaneous thoracolumbar pedicle screw placement. Technical note, Journal of neurosurgery 99(3 Suppl) (2003) 324-9.

[12] S.A. Klein, S.D. Glassman, J.R. Dimar, 2nd, M.J. Voor, Evaluation of the fixation and strength of a "rescue" revision pedicle screw, Journal of spinal disorders \& techniques 15(2) (2002) $100-4$.

[13] A. Bertelsen, J. Melo, E. Sanchez, D. Borro, A review of surgical robots for spinal interventions, The international journal of medical robotics + computer assisted surgery : MRCAS 9(4) (2013) 407-22.

\section{Disclosures}

The authors have no conflict of interest to disclosure. 